\title{
Modeling Idea Markets: Between Beauty Contests and Prediction Markets
}

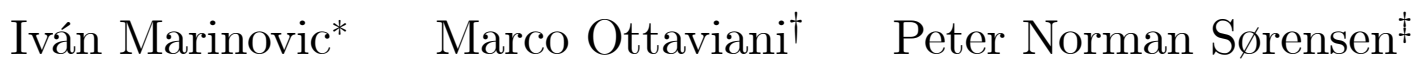

September 2010

\section{Introduction}

This paper is motivated by a recent surge of interest in evaluating ideas (such as new products or corporate projects) by means of trading mechanisms known as idea markets. In idea markets, ideas are associated to assets that are then traded among market participants who possess relevant information. In a baseline design, traders are rewarded on the basis of the value of their portfolio when the market is closed. ${ }^{1}$ Idea markets hold great promise to initially complement and eventually substitute traditional techniques for market research mostly based on costly surveys. In this paper, we investigate the information aggregation properties of idea markets, focusing on the role played by the incentive to buy popular assets, and on how this incentive depends on the size of the market.

Proposers of idea markets are inspired by the performance of prediction markets, such as Iowa electronic markets as well as horse race betting markets. ${ }^{2}$ A defining feature of

*Kellogg School of Management, Northwestern University, 2001 Sheridan Road, Evanston, IL 60208-2013. Phone: +1-847-491-2677. E-mail: i-marinovic@kellogg.northwestern.edu. Web: http://www.kellogg.northwestern.edu/accounting/doctoral/students.htm.

${ }^{\dagger}$ Kellogg School of Management, Northwestern University, 2001 Sheridan Road, Evanston, IL 602082013. Phone: +1-847-467-0684. Fax: +1-847-467-1777. E-mail: m-ottaviani@northwestern.edu. Web: http://www.kellogg.northwestern.edu/Faculty/ottaviani/homepage/.

$\ddagger$ Department of Economics, University of Copenhagen, Øster Farimagsgade 5, Building 26, DK-1353 Copenhagen K, Denmark. Phone: +45-3532-3056. Fax: +45-3532-3000. E-mail: peter.sorensen@ econ.ku.dk. Web: http://www.econ.ku.dk/sorensen.

${ }^{1}$ See Plott and Chen's (2002) pioneering experiment. For a brief introduction to the topic we refer to Ottaviani (2009).

${ }^{2}$ See Wolfers and Zitzewitz (2005) for an introduction to prediction markets. See Berg and Rietz (2006) and Forsythe et al. (2008) on the Iowa Electronic Markets. See Ottaviani and Sørensen (2009) and (2010) and references therein on horse-race betting. 
prediction markets is that assets are liquidated on the basis of the ex-post realization of the underlying variable. Thus, traders in prediction markets have an incentive to maximize the value of their portfolio by picking the assets they deem to be most valuable. The resulting equilibrium price in a prediction market should then reflect the traders' overall information, very much like in a financial market driven by fundamentals. ${ }^{3}$

While participants in prediction markets gain from predicting accurately the final outcome, in idea markets participants are rewarded on their ability to accurately predict the choices of other market participants. ${ }^{4}$ This is often because the ex post performance of the underlying ideas is difficult to observe or verify. ${ }^{5}$ To wit, the profitability of the ideas that are not implemented is simply not observed. And even when information about the profitability of an implemented idea is observed, typically this is only with sizeable noise and major delay. Without ex post validation that is characteristic of prediction markets, pure idea markets become beauty contests.

This paper investigates how much information is aggregated when agents thus face mixed rewards based partly on ex-post realizations (as is typical of prediction markets) and partly on the most popular opinion (as in a beauty contest). As a first attempt to formally model this tension, we analyze a variant of a model proposed by Morris and Shin (2002). The model features a set of agents endowed with both private and common information who simultaneously submit predictions. With some probability agents are rewarded on the basis of the accuracy of their individual prediction relative to the final outcome. With complementary probability, agents are rewarded on the distance between their prediction and the average prediction across agents (the consensus forecast). This probabilistic interpretation of the rewards captures the notion that the market designer may only observe the value of the idea with some probability, which we refer to as the prediction market intensity.

The mixed nature of rewards means that agents care not only about their own assessment of the final outcome but also - because of the incentives to coordinate with other agents - about their assessments of other agents' assessments, and about the assessments

\footnotetext{
${ }^{3}$ See Hayek's (1945) classic essay about the ability of markets to aggregate dispersed information, Grossman's (1976) theoretical development of the concept of rational expectation equilibrium, and Surowiecki's (2004) popularization of how markets can harness the wisdom of the crowds.

${ }^{4}$ See Spears et al. (2009)

${ }^{5}$ Wolfers and Zitzewitz (2006) also stress that the key variables of interest are often difficult to write into contracts.
} 
of other agents about the assessments of other agents, and so on. Higher order beliefs play a key role in these markets, as in Keynes's (1936) celebrated metaphor of financial markets as beauty contests. ${ }^{6}$

We examine how these beauty contest incentives affect the informativeness of the consensus forecast. We begin by considering pure markets, where agents only care about one type of incentive. A pure idea market is a beauty contest in which agents are only driven by the incentive to second guess other agents' beliefs so as to meet the consensus forecast. As a result, agents rely exclusively on common information, which is the most effective way to coordinate with other agents. But precisely because agents disregard private information, the informativeness of the consensus forecast in a beauty contest becomes very poor. ${ }^{7}$

In the opposite extreme case of a pure prediction market, the consensus forecast aggregates information much more effectively. An agent's optimal strategy consists of submitting a prediction that coincides with the agent's best predictor of the idea's value, which results in a consensus forecast that at least to some extent reflects agents' private information. Aggregating the private information, the consensus forecast is more accurate the greater is the number of agents in the prediction market. However, the consensus forecast still overweights the common information relative to an optimal aggregate of all private and public information. As a result, the informativeness of the consensus forecast need not increase in the precision of common information. An increase in the precision of common information has two effects. Holding agents' strategies constant, more precise common information leads naturally to a more precise consensus forecast. These informational benefits, though, may be offset by agents' reaction: a more precise common signal

\footnotetext{
${ }^{6}$ See Keynes (1936), page 156: “... professional investment may be likened to those newspaper competitions in which the competitors have to pick out the six prettiest faces from a hundred photographs, the prize being awarded to the competitor whose choice most nearly corresponds to the average preferences of the competitors as a whole; so that each competitor has to pick, not those faces which he himself finds prettiest, but those which he thinks likeliest to catch the fancy of the other competitors, all of whom are looking at the problem from the same point of view. It is not a case of choosing those which, to the best of one's judgement, are really the prettiest, nor even those which average opinion genuinely thinks the prettiest. We have reached the third degree where we devote our intelligences to anticipating what average opinion expects the average opinion to be. And there are some, I believe, who practise the fourth, fifth and higher degrees."

${ }^{7}$ In a pure idea market, we find that there is a unique equilibrium in linear strategies, contrary to conventional wisdom. Multiplicity arises only when agents have common knowledge about fundamentals. But the slightest breakdown in agents' common knowledge results in a unique equilibrium, suggesting that multiplicity of equilibria is a knife edge result.
} 
induces agents to increase the weight they attach to the common signal at the expense of the private signal. Since the consensus forecast assigns excessive weight on common information, this effect leads to a redundancy of common information in the consensus forecast, eventually reducing its informativeness.

After reviewing the two pure cases, we examine the case in which agents have mixed incentives. Intuitively, in equilibrium, agents' predictions are a weighted average of their private and common signals. First and foremost, we establish that the informativeness of the consensus forecast increases in the intensity of prediction markets. By reducing agents' coordination concerns, a greater intensity of prediction markets lowers the weight of common information in agents' predictions, thereby reducing the redundancy of common information that affects the consensus forecast.

What is the impact of an increase in the number of (privately informed) agents on the informativeness of the consensus forecast? Our main result is a characterization of situations in which an increase in the number of agents has a detrimental effect on the informativeness of the consensus forecast. As we show, increasing the number of agents has two effects. On the one hand, there is the statistical benefit of a larger sample - more agents allow the consensus forecast to filter more efficiently the noise of agents' private signals. On the other hand, a larger sample has also an indirect effect: the distortions that arise from agents' coordination incentives are worsened when there is a larger number of informed agents. In a smaller sample, the influence of an individual agent over the consensus forecast is larger, so that each agent is less concerned in second guessing the predictions of other agents. As the number of agents grows, the impact of any given agent on the consensus forecast decreases. Coordination becomes then a more important concern for an agent. And agents' greater emphasis on coordination lowers the informativeness of the consensus forecast, even to the point of offsetting the statistical benefits of a larger sample.

We also find, in the general case, that better common information may deteriorate the informativeness of the consensus forecast. ${ }^{8}$ The mechanism is similar to that described for a pure prediction market, but aggravated here by agents' coordination incentives. An

\footnotetext{
${ }^{8}$ This is a feature also stressed by Morris and Shin (2002). However, by focusing on a version of the model with a continuum of agents, Morris and Shin do not analyze how information aggregation depends on the prediction market intensity and the number of informed agents. Instead, we focus on these questions that are natural in the context of our interpretation in terms of idea markets.
} 
increase in the quality of common information not only helps agents to make better predictions but also helps them to coordinate more efficiently. Agents respond by relying more heavily on common information when they form their predictions. Consequently, the correlation between the consensus forecast and agents' private signals becomes weaker, particularly when the number of agents is large. This in turn lowers the informational content of the consensus when private signals are more accurate than common information.

Other theoretical work on prediction markets addresses different problems. Hanson and Oprea (2009) and Hahn and Tetlock (2007) analyze the incentives of prediction market agents to affect the market price in order to affect the decision based on that price. Ottaviani and Sørensen (2007) investigate the problem of outcome manipulation whereby agents affect the outcome on which the prediction market payoff is paid. Lieli and Nieto Barthaburu (2009) analyze the effect of feedback on the operation of prediction markets when a decision maker's intervention, based on the information revealed by the market, affects the probability of the underlying event.

We proceed by describing the model in Section 2. In Section 3 we introduce a notion of informativeness. In Section 4 we characterize the equilibrium and discuss its informational properties.

\section{Model}

The model is a variant of Morris and Shin (2002) with a finite number of agents rather than a continuum of agents.

A market designer is interested in learning the value of an idea, which is captured by the state $\theta$. The prior of the state follows an improper uniform distribution over the real line. ${ }^{9}$ There is a group of agents, indexed by $i \in\{1,2, . . n\}$, who are privately and heterogeneously informed about $\theta$. Agents' information set consists of two signals: (i) a common signal, that is observed by all agents, which we represent by the random variable $y=\theta+\eta$ and (ii) a private signal $x_{i}=\theta+\varepsilon_{i}$ which is only observed by agent $i$. We assume that the error terms $\left\{\left\{\varepsilon_{i}\right\}_{i=1}^{n}, \eta\right\}$ are mutually independent and jointly normally distributed with $\eta \sim N\left(0, \frac{1}{\alpha}\right)$ and $\varepsilon_{i} \sim N\left(0, \frac{1}{\beta}\right)$.

\footnotetext{
${ }^{9}$ The assumption that $\theta$ is uniformly distributed on the real line is non standard but presents no technical difficulties as long as we are concern with conditional beliefs. Morris and Shin (2003) argue that this assumption can be considered as the limiting case as the prior distribution of $\theta$ becomes diffuse.
} 
The market designer observes neither the common nor the private signals. To collect this information, the market designer asks each agent to make a sealed prediction about $\theta$, so that agents simultaneously and independently predict the value of $\theta$. In exchange, the market designer commits to reward agents according to a publicly announced reward rule that defines how each agent is rewarded based on the prediction made, the realization of $\theta$, and the predictions of other agents. In particular, let $a_{i}$ be agent $i$ 's action or prediction. The payoff of agent $i$ who predicted $a_{i}$ when the state of nature is $\theta$ and the opponents' predictions are $\mathbf{a}_{-i} \equiv\left\{a_{1}, \ldots, a_{i-1}, a_{i+1}, \ldots, a_{n}\right\}$ is given by

$$
u_{i}\left(\theta, \mathbf{a}_{-i}, a_{i}\right) \equiv-\delta\left(a_{i}-\theta\right)^{2}-(1-\delta)\left(a_{i}-\bar{a}_{n}\right)^{2}
$$

where $\bar{a}_{n}=\frac{a_{1}+a_{2}+\ldots+a_{n}}{n}$ is the average prediction among all agents which we refer to as the consensus forecast. We assume that agents are risk neutral, maximizing the expected payoff.

This reward rule has two components:

- The first component, the accuracy term, $\left(a_{i}-\theta\right)^{2}$, is a standard quadratic loss in the distance between the underlying state and the action. This payoff component represents the cost of forecast error. Like in prediction markets, this component induces agents to anchor their predictions on the fundamentals $\theta{ }^{10}$

- The second component, the beauty contest term, $-\left(a_{i}-\bar{a}_{n}\right)^{2}$, represents the cost from being away from the consensus forecast. Like in idea markets, this term introduces a coordination motive or beauty contest incentive that induces an agent to second guess the opponents' beliefs. ${ }^{11}$

In the sequel, we refer to $\delta$ (respectively, $1-\delta$ ) as the prediction market intensity (respectively, idea market intensity). We refer to the case $\delta=1$ as a pure prediction market and

\footnotetext{
${ }^{10}$ This is a reduced-form model of a market, but we may imagine agent $i$ adopting a position in a risky asset. The ideal position depends on the parameter $\theta$ (or $\bar{a}_{n}$ if the designer substitutes that for $\theta$ in order to close market positions). By optimality, there is a negligible first-order loss of trading as if the parameter is equal to $a_{i}$, and our payoff expression $-\left(a_{i}-\theta\right)^{2}$ captures the second-order effect.

${ }^{11}$ The fact that agents have a coordination motive means that their actions are strategic complements. Note, however, that trading in a financial market might induce strategic substitutability. Also, the incentive to be close to the consensus is opposite to the incentive to differentiate one's forecast in a large winner-takes-all forecasting contest (see Ottaviani and Sørensen, 2006). To properly illustrate the consequences of a dominant beauty contest effect, here we focus on the case where the total effect results in strategic complements.
} 
$\delta=0$ as a pure idea market. ${ }^{12}$

The reward rule (1) can also be interpreted probabilistically, in which case $\delta$ represents the probability the market designer observes the value of $\theta$ and rewards agents on that basis and $1-\delta$ represents the probability that the designer does not observe $\theta$ and thus rewards agents on the basis of the consensus forecast. ${ }^{13}$

The structure of the game is common knowledge. When all agents use symmetric linear forecasting rules, as in all equilibria discussed below, the designer's best estimate of the location of $\theta$ is the consensus forecast $\bar{a}_{n}$.

\section{Informativeness of Idea Markets}

Our purpose is to characterize the determinants of the informational properties of the consensus forecast $\bar{a}_{n}$. In our setting with normally distributed posterior beliefs on $\theta$, a natural measure of market informativeness is given by the precision of the posterior distribution of the state conditional on the consensus forecast, $\gamma=1 / \operatorname{var}\left(\theta \mid \bar{a}_{n}\right)$. The value of $\gamma$ is bounded above by $\alpha+n \beta$ which results in the most informative scenario where the prior uncertainty about $\theta$ is reduced both by knowledge about the common signal $y$ and also by all agents' private signals $\mathbf{x} \equiv\left\{x_{i}\right\}_{i=1}^{n}$.

We proceed by first considering the two polar cases of a pure idea market $\delta=0$ and a pure prediction market $\delta=1$.

\subsection{Pure Idea Market}

Idea markets may suffer from multiplicity of equilibria arising from agents' incentives to coordinate their actions. Restricting attention to symmetric equilibria where strategies are linear in the common and private signals, this multiplicity of equilibria is only present when private signal errors $\varepsilon_{i}$ are imperfectly correlated. For instance, when agents' private signals are perfect, they are indifferent between coordinating through the common signal (i.e., $a_{i}=y$ ) or through the private signals (i.e., $a_{i}=x_{i}=\theta$ ). In fact, they are indifferent between either of these pure alternatives and any possible combination of $y$ and $x_{i}$. But,

\footnotetext{
${ }^{12}$ Of course, this simple reward rule is somewhat arbitrary, may not even be optimal, but provides a simple setting to compare the informational properties of prediction and idea markets.

${ }^{13}$ In general, one would think that $\delta$ is endogenous as it depends on whether or not the market designer undertakes the idea. This is perhaps an interesting extension.
} 
in the general case when private signal are imperfectly correlated, agents must disregard their private information in equilibrium.

Proposition 1 When $\delta=0$, in any symmetric equilibrium in linear strategies, agents put no weight on their private signal. Hence, $\gamma=1 / \operatorname{var}\left(\theta \mid \bar{a}_{n}\right) \leq 1 / \operatorname{var}(\theta \mid y)=\alpha$.

The proposition follows as a corollary to Proposition 4 below. A pure idea market is thus very uninformative because agents' actions only convey common information which implies that all the information contained in private signals is lost. In a pure idea market, the coordination motive overwhelms agents' actions; an agent's prediction is chosen not on the basis of fundamentals but only on the basis of the expected actions by the opponents.

\subsection{Pure Prediction Market}

In a pure prediction market there is a linear equilibrium in which agents submit their best linear predictor of $\theta$.

Proposition 2 When $\delta=1$, there is a unique symmetric equilibrium in linear strategies in which $a_{i}=E\left(\theta \mid y, s_{i}\right)=\frac{\alpha y+\beta x_{i}}{\alpha+\beta}$ for all $i=1, . ., n$. In this equilibrium, informativeness is given by $\gamma(\delta=1)=\frac{n(\alpha+\beta)^{2}}{\alpha n+\beta}$.

Proof. The consensus forecast is given by $\bar{a}_{n}=\frac{\alpha y+\beta \bar{x}_{n}}{\alpha+\beta}$ where $\bar{x}_{n}=\frac{x_{1}+\ldots+x_{n}}{n}$, so that $\operatorname{var}\left(\bar{a}_{n} \mid \theta\right)=\left(\frac{1}{\alpha+\beta}\right)^{2}\left(\frac{\alpha^{2}}{\alpha}+\frac{\beta^{2}}{n^{2}} \frac{n}{\beta}\right)=\frac{\alpha n+\beta}{(\alpha+\beta)^{2} n}$. Given that the prior is improper, we have $\operatorname{var}\left(\theta \mid \bar{a}_{n}\right)=\operatorname{var}\left(\bar{a}_{n} \mid \theta\right)$ (see Hartigan, 1983) and thus we obtain the expression for the informativeness $\gamma(\delta=1)$.

In pure prediction markets an agent's prediction is his individually most efficient estimate of the fundamentals $\theta$. The consensus forecast is a weighted average of the common signal $y$ and the sufficient statistic $\bar{x}_{n}$ for the private signals. As is well known, the consensus forecast puts greater weight on the common signal $y$ than would an optimal estimate $E\left(\theta \mid y, \bar{x}_{n}\right)=\frac{\alpha y+n \beta \bar{x}_{n}}{\alpha+n \beta}$ for $\theta$ based on $y$ and $\bar{x}_{n}$.

The precision of this consensus estimate is always favored by the precision of both common and private information. Despite this, the informativeness of the consensus forecast may decrease in the precision of common information. 
Corollary 3 When $\delta=1$, informativeness increases in $\beta$ and in $n$ but decreases in the precision of the common signal if and only if $(\alpha-\beta) n+2 \beta \leq 0$.

Proof. From the expression in Proposition 2, we have $\frac{\partial \gamma(\delta=1)}{\partial \beta}=\frac{n(\alpha+\beta)[(2 n-1) \alpha+\beta]}{(\alpha n+\beta)^{2}}, \frac{\partial \gamma(\delta=1)}{\partial \alpha}=$ $\frac{n(\alpha+\beta)[n \alpha+(2-n) \beta]}{(\alpha n+\beta)^{2}}$, and $\frac{\partial \gamma(\delta=1)}{\partial n}=\frac{\beta(\alpha+\beta)^{2}}{(\alpha n+\beta)^{2}}$.

The excessive weight accorded by the consensus forecast to the common signal relative to the optimal predictor is particularly great when the number of agents is large. A higher $\alpha$ can aggravate this problem. If we held agents' strategies constant, an increase in $\alpha$ would always result in a more informative consensus forecast, simply because the consensus forecast would contain less noise. But an increase in $\alpha$ induces agents to assign additional weight to the common signal $y$, thereby reducing even more the correlation between the consensus forecast and agents' private signals. This effect would never reduce the informativeness of the consensus forecast when the common signal is more precise than the private signal but it does so when the opposite is true.

\section{Mixed Incentives}

The polar cases of pure markets discussed above serve as benchmarks. We now turn to the more interesting case in which agents have a mix of prediction market and idea market incentives, so that $\delta \in(0,1)$. For convenience, we use the short-hand notation $E_{i}(\cdot)=E_{i}\left(\cdot \mid y, x_{i}\right)$.

Agent i's optimization program is to solve

$$
\max _{a} E_{i}\left[-\delta(a-\theta)^{2}-(1-\delta)\left(a-\bar{a}_{n}\right)^{2}\right]
$$

and the agent's optimal action is characterized by the first-order condition

$$
a_{i}=\delta E_{i}(\theta)+(1-\delta) E_{i}\left(\bar{a}_{n}\right)
$$

Intuitively, equation (2) shows that agent i's optimal prediction is a weighted average of the assessment of the fundamentals and of the consensus forecast. To estimate the consensus forecast, an agent must forecast the forecasts of others, as in Townsend (1983).

To solve this problem, we first assume (and then verify) the existence of a symmetric linear equilibrium in which an agent's strategy is a weighted average of private and common 
signals, as described by

$$
a_{i}=\varphi y+(1-\varphi) x_{i}
$$

where $\varphi$ is the weight attached to the common signal in an agent's equilibrium prediction. According to this candidate equilibrium, the agent expects the consensus forecast to be given by

$$
E_{i}\left(\bar{a}_{n}\right)=\frac{a_{i}+(n-1) E_{i}\left(a_{-i}\right)}{n},
$$

where $a_{-i}$ is the prediction of any opponent of agent $i$. Substituting $E_{i}\left(\bar{a}_{n}\right)$ into equation $(2)$, we find

$$
a_{i}=\frac{\delta E_{i}(\theta)+(1-\delta) \frac{(n-1)}{n} E_{i}\left(a_{-i}\right)}{1-\frac{(1-\delta)}{n}},
$$

and, using (3), we obtain

$$
a_{i}=\frac{\delta \frac{\alpha y+\beta x_{i}}{\alpha+\beta}+(1-\delta) \frac{(n-1)}{n}\left[\varphi y+(1-\varphi) \frac{\alpha y+\beta x_{i}}{\alpha+\beta}\right]}{1-\frac{(1-\delta)}{n}}
$$

Finally, comparing coefficients in (3) and (4) yields

$$
\varphi=\frac{(n-1+\delta) \alpha}{(n-1+\delta) \alpha+n \delta \beta},
$$

thus establishing the following result:

Proposition 4 For all $\delta \in[0,1]$, there exists a unique symmetric equilibrium in linear strategies $a_{i}=\varphi y+(1-\varphi) x_{i}$, where

$$
\varphi=\frac{(n-1+\delta) \alpha}{(n-1+\delta) \alpha+n \delta \beta} .
$$

To introduce the properties of the equilibrium we first consider two limit cases in terms of the number of participants in the market. When the number of agents grows large, the weight attached to the common signal in agents' equilibrium strategy converges to $\lim _{n \rightarrow \infty} \varphi=\frac{\alpha}{\alpha+\delta \beta}$, which is larger than the weight prescribed by the individually optimal linear predictor, $\frac{\alpha}{\alpha+\beta}$. By contrast, when there is only one agent, the weight attached to the common signal coincides with that of the optimal linear prediction, $\lim _{n \rightarrow 1} \varphi=$ $\frac{\alpha}{\alpha+\beta}$. Intuitively, these cases suggest that agents tend to assign an excessive weight to the common signal when they have coordination concerns. The next corollary studies the determinants of $\varphi$. 
Corollary 5 The weight $\varphi$ attached to the common signal y in agents' equilibrium strategies decreases in the intensity of prediction markets $\delta$ and in the precision of the private signal $\beta$ and increases in the number of agents $n$ and in the precision of the common signal $\alpha$.

Proof. We have $\frac{\partial \varphi}{\partial \delta}=\frac{-n(n-1) \alpha \beta}{[(n-1+\delta) \alpha+n \delta \beta]^{2}}<0$ for all $n>1, \frac{\partial \varphi}{\partial n}=\frac{\delta(1-\delta) \alpha \beta}{[(n-1+\delta) \alpha+n \delta \beta]^{2}}>0$ for $\delta \in(0,1), \frac{\partial \varphi}{\partial \alpha}=\frac{n(n-1+\delta) \delta \beta}{[(n-1+\delta) \alpha+n \delta \beta]^{2}}>0$, and $\frac{\partial \varphi}{\partial \beta}=\frac{-(n-1+\delta) n \delta \alpha}{[(n-1+\delta) \alpha+n \delta \beta]^{2}}<0$.

The effect of $\alpha$ and $\beta$ on $\varphi$ are intuitive: the weight attached to the common signal increases in the relative precision of the common signal.

The other two effects are more central to our central questions regarding idea markets. Agents' coordination incentives become stronger when the intensity $\delta$ of prediction markets is lower, or when the size $n$ of the market is larger. To the extent that agents are more concerned about coordination they increase the weight of the common signal. This weight is excessive relative to the optimal predictor of $\theta$ already in the pure prediction market, and the effect is only aggravated when more weight is accorded to the idea market.

\subsection{Informativeness of Consensus Forecast}

In this section we examine the determinants of informativeness. First we consider how the precision of agents' signals affect informativeness and then we study how the intensity of prediction markets and the number of agents affect informativeness. We show that both the precision of common information and the number of agents may be detrimental to informativeness. As expected, we verify that the intensity of prediction markets enhances the informativeness of the consensus forecast.

Our analysis relies on the following intermediate result:

Lemma 6 The posterior belief $\theta \mid \bar{a}_{n} \sim N\left(\bar{a}_{n}, \operatorname{var}\left(\theta \mid \bar{a}_{n}\right)\right)$ where

$$
\operatorname{var}\left(\theta \mid \bar{a}_{n}\right)=\frac{\varphi^{2}}{\alpha}+\frac{(1-\varphi)^{2}}{n \beta}=\frac{(n-1+\delta)^{2} \alpha+n \delta^{2} \beta}{[(n-1+\delta) \alpha+n \delta \beta]^{2}} .
$$

Proof. Since $\bar{a}_{n}=\frac{a_{1}+\ldots+a_{n}}{n}=\varphi y+(1-\varphi) \frac{x_{1}+\ldots+x_{n}}{n}$, we have $\bar{a}_{n} \mid \theta \sim N\left(\theta\right.$, var $\left.\left(\bar{a}_{n} \mid \theta\right)\right)$ with $\operatorname{var}\left(\bar{a}_{n} \mid \theta\right)=\varphi^{2} \frac{1}{\alpha}+(1-\varphi)^{2} \frac{n}{n^{2} \beta}$. It follows from Hartigan (1983) that the posterior is as claimed with $\operatorname{var}\left(\theta \mid \bar{a}_{n}\right)=\operatorname{var}\left(\bar{a}_{n} \mid \theta\right)$. The second part of equation (6) follows from (5). 
Armed with this lemma we now turn the determinants of informativeness. We begin by investigating the impact of the most direct determinants of informativeness, the precision of the signals.

Proposition 7 (i) Informativeness increases with the precision of the private signal $\beta$; (ii) Informativeness increases with the precision of the common signal $\alpha$ if and only if $\alpha / \beta \geq \frac{(n-1-\delta) n \delta}{(n-1+\delta)^{2}}$.

Proof. For part (i) we use (6) to find $\frac{\partial \operatorname{var}\left(\theta \mid \bar{a}_{n}\right)}{\partial \beta}=-n \delta \frac{\left[\delta(\delta+3 n-3)+2(n-1)^{2}\right] \alpha+n \delta^{2} \beta}{[(n-1+\delta) \alpha+n \delta \beta]^{3}}<0$. For part (ii), we can likewise derive $\frac{\partial \operatorname{var}\left(\theta \mid \bar{a}_{n}\right)}{\partial \alpha}=\frac{(n-1+\delta)\left[(n-1-\delta) n \delta \beta-(n-1+\delta)^{2} \alpha\right]}{[(n-1+\delta) \alpha+n \delta \beta]^{3}}$. Its sign depends on the factor $(n-1-\delta) n \delta \beta-(n-1+\delta)^{2} \alpha$, as stated.

Already Corollary 3 established that even in a pure prediction market a more precise common signal may reduce informativeness, simply because the consensus forecast may end up capturing too little of the information contained in the private signals. The presence of idea market incentives should, if anything, aggravate this problem because agents' coordination incentives induce them to overweight the common signal thereby increasing the redundancy of common information in the consensus forecast.

Consider the effect of the intensity of idea markets on informativeness.

Proposition 8 Informativeness decreases in the intensity of idea markets: $\frac{\partial \operatorname{var}\left(\theta \mid \bar{a}_{n}\right)}{\partial \delta}>0$.

Proof. Note that $\operatorname{var}\left(\theta \mid \bar{a}_{n}\right)=\frac{\varphi^{2}}{\alpha}+\frac{(1-\varphi)^{2}}{n \beta}$ is a convex function of $\varphi$ which has a unique minimum at $\underline{\varphi}=\frac{\alpha}{\alpha+n \beta}$, and therefore is increasing for $\varphi>\underline{\varphi}$. By (5), $\varphi>\underline{\varphi}$ is equivalent to $(n-1+\delta)>\delta$, which is true. From Corollary $5, \frac{\partial \varphi}{\partial \delta}<0$, so we can conclude that $\operatorname{var}\left(\theta \mid \bar{a}_{n}\right)$ increases with $\delta$.

The reason that the intensity of idea markets hampers the informational content of the consensus forecast should not be surprising. The presence of idea market incentives excessively reduces the correlation between agents' predictions and agents' information about fundamentals. A clear implication from this observation is that a market designer with discretion over the value of $\delta$ might want to set $\delta=1$ in order to maximize the informational content of the consensus forecast. We conclude that a designer who always observe the value of the fundamentals, would optimally organize this market as a pure prediction market. 
The informational effect of $n$ is less intuitive and perhaps more surprising. Increasing the number of agents has two effects. On the one hand, there are the traditional statistical benefits of a larger sample: holding agents' strategies constant, an increase in the number of agents allows the consensus forecasts to filter more efficiently the noise included in agents' private signals. We call this the large sample effect. On the other hand, as the number of agents increases, agents are induced to strategically increase the weight they assign to the common signal, thereby reducing the informational content of the consensus forecast. This is the second guessing effect. Remarkably, under certain circumstances the second guessing effect overwhelms the large sample effect so that increasing $n$ decreases the informational content of $\bar{a}_{n}$.

Proposition 9 For sufficiently small prediction market intensity, $\delta<\delta^{*}=\frac{\sqrt{9 \alpha^{2}+8 \alpha \beta}-3 \alpha}{2 \beta} \in$ $(0,1)$, informativeness is a single-peaked function of the number of agents $n$, decreasing when $n>n^{*}=\frac{(1-\delta)(2-\delta) \alpha}{(2-3 \delta) \alpha-\delta^{2} \beta}$. When instead $\delta \geq \delta^{*}$, informativeness increases in $n$.

Proof. From (6), we have $\frac{\partial \operatorname{var}\left(\theta \mid \bar{a}_{n}\right)}{\partial n}=\beta \delta \frac{(\delta-1)(2-\delta) \alpha+\left[(2-3 \delta) \alpha-\delta^{2} \beta\right] n}{[(n-1+\delta) \alpha+n \delta \beta]^{3}}$. Its sign depends on $A=$ $(\delta-1)(2-\delta) \alpha+\left[(2-3 \delta) \alpha-\delta^{2} \beta\right] n$, which has a negative intercept, $(\delta-1)(2-\delta) \alpha<$ 0 . The slope $\frac{\partial A}{\partial n}=(2-3 \delta) \alpha-\delta^{2} \beta$ is a concave function of $\delta$ and takes the value of $2 \alpha>0$ when $\delta=0$ and the value $-(\alpha+\beta)<0$ when $\delta=1$. Given that $\frac{\partial A}{\partial n}$ attains its maximum at $\delta=-\frac{3 \alpha}{2 \beta}<0$ and decreases in $\delta$ over $[0,1]$, there exists $\delta^{*} \in(0,1)$ such that $\frac{\partial A}{\partial n} \leq 0$ if and only if $\delta \geq \delta^{*}$, as stated. Combining this fact with the earlier observation the intercept is negative, we conclude that $A<0$ if $\delta \geq \delta^{*}$. By contrast, $\frac{\partial A}{\partial n}>0$ if $\delta<\delta^{*}$, in which case $A \geq 0$ if and only if $n \geq n^{*}$, as stated.

Figure 1 plots informativeness $\gamma$ as a function of the number of agents $n$. There we see that a larger sample of agents increases the information content of the consensus forecast when the intensity of idea markets is low, as illustrated by the solid curve. By contrast, a larger sample aggravates the second guessing effect when the intensity of idea markets is high, as in the dashed and dotted curves. This is remarkable, because it means that the second guessing effect associated with the intensity of idea markets may destroy a lot of information, even reversing the benefits of a larger sample of agents. 
Figure 1: Illustration of the impact of the number of agents $n$ on informativeness $\gamma$ for $\alpha=\beta=1$. The curves correspond to $\delta=0.8>\delta^{*}$ (solid), $\delta=0.5<\delta^{*}$ (dashed, with corresponding $n^{*}=3$ ), and $\delta=0.1$ (dotted, with corresponding $n^{*} \approx 1$ ). 


\section{Conclusion}

In this paper we characterize the informational properties of the consensus forecast when agents trade off the incentive to accurately predict fundamentals (as in pure prediction markets) with the desire of meeting the most popular opinion (as in pure idea markets, or beauty contests).

The main lesson of our analysis is that many of the intuitive properties that usually characterize standard markets or even prediction markets do not hold when agents have coordination motives, as in idea markets. For example neither a larger sample of agents nor a better quality of common information are necessarily beneficial in terms of the informational properties of the market. The design of an idea market is thus a delicate task.

A key limitation of our analysis is that it relies on reduced-form rewards, in keeping with the literature on beauty contests. A crucial assumption in our model is that agents' actions are strategic complements. It would be interesting to analyze idea markets in a fully micro-founded model of trading. We expect our results to be affected if we instead assumed that agents' actions are strategic substitutes (see Angeletos and Pavan, 2007), as in financial markets where traders have market power à la Kyle (1985).

\section{References}

Angeletos, George-Marios, and Alessandro Pavan (2007), "Efficient Use of Information and Social Value of Information," Econometrica, 75(4), 1103-1142.

Berg, Joyce E., and Thomas A. Rietz (2006), "The Iowa Electronic Markets: Stylized Facts and Open Issues." In Information Markets: A New Way of Making Decisions, edited by Robert W. Hahn and Paul C. Tetlock. AEI-Brookings Joint Center for Regulatory Studies.

Forsythe, Robert, Forrest Nelson, George R. Neumann, and Jack Wright (1992), "Anatomy of an Experimental Political Stock Market," American Economic Review, 82(5), $1142-1161$.

Grossman, Sanford J. (1976), "On the Efficiency of Competitive Stock Markets Where Traders Have Diverse Information." Journal of Finance, 31(2), 573-585. 
Hahn, Robert W., and Paul C. Tetlock (2007), "Optimal Liquidity Provision for Decision Makers." Working Paper, University of Texas at Austin and AEI-Brookings Joint Center for Regulatory Studies.

Hanson, Robin and Ryan Oprea (2009), "A Manipulator Can Aid Prediction Market Accuracy," Economica, 76(302), 304-314.

Hayek, Friedrich A. (1945), "The Use of Knowledge in Society," American Economic Review, 35(4), 519-530.

Hartigan, John (1983), Bayes Theory. Springer, New York.

Keynes, John Maynard (1936), The General Theory of Employment, Interest and Money. Macmillan, London.

Kyle, Albert S. (1985), "Continuous Auctions and Insider Trading," Econometrica, 53(6), $1315-1335$.

Morris, Stephen and Hyhn Song Shin (2002), "Social Value of Public Information," American Economic Review, 92(5), 1521-1534.

Lieli, Robert P. and Augusto Nieto-Barthaburu (2009), "Feedback in Prediction Markets." Working Paper, University of Texas at Austin and

East Carolina University.

Ottaviani, Marco (2009), "The Design of Idea Markets: An Economist's Perspective," Journal of Prediction Markets, 3(1), 41-43.

Ottaviani, Marco, and Peter Norman Sørensen (2006), "The Strategy of Professional Forecasting," Journal of Financial Economics, 81(2), 441-466.

Ottaviani, Marco, and Peter Norman Sørensen (2007), "Outcome Manipulation in Corporate Prediction Markets," Journal of the European Economic Association, Papers and Proceedings, 5(2-3), 554-563.

Ottaviani, Marco, and Peter Norman Sørensen (2009), "Surprised by the Parimutuel Odds?" American Economic Review, 99(5), 2129-2134. 
Ottaviani, Marco, and Peter Norman Sørensen (2010), "Noise, Information, and the Favorite-Longshot Bias in Parimutuel Predictions," American Economic Journal: Microeconomics, 2(1), 58-85.

Plott, Charles, and Kay-Yut Chen (2002), "Information Aggregation Mechanisms: Concepts, Design and Implementation for a Sales Forecasting Problem," Social Science Working Paper 1131, California Institute of Technology.

Spears, Brian, Christina LaComb, John Interrante, Janet Barnett, and Deniz SenturkDogonaksoy (2009), "Examining Trader Behavior in Idea Markets: An Implementation of GE's Imagination Markets," Journal of Prediction Markets, 3(1), 17-39.

Surowiecki, James (2004), The Wisdom of Crowds. Doubleday Publishing, New York.

Townsend, Robert M. (1983), "Forecasting the Forecasts of Others," Journal of Political Economy, 91(4), 546-588.

Wolfers, Justin, and Eric Zitzewitz (2004), "Prediction Markets," Journal of Economic Perspectives, 18(2), 107-126.

Wolfers, Justin, and Eric Zitzewitz (2006), "Five Open Questions About Prediction Markets," In Information Markets: A New Way of Making Decisions, edited by Robert W. Hahn and Paul C. Tetlock. AEI-Brookings Joint Center for Regulatory Studies. 